\title{
Spectrométrie Raman de résonance picoseconde : principe et application à l'analyse structurale d'intermédiaires réactionnels en solution
}

\author{
V. De Waele, O. Poizat et G. Buntinx
}

\begin{abstract}
Laboratoire de Spectrochimie Infrarouge et Raman (LASIR), UMR 8516, Centre d'Études et de Recherches Lasers et Applications, Université des Sciences et Technologies de Lille, batiment $C_{5}, 59655$ Villeneuve-d'Ascq cedex, France
\end{abstract}

\begin{abstract}
Résumé : La spectrométrie Raman de résonance picoseconde est une technique de choix pour analyser les états excités et intermédiaires réactionnels impliqués dans des processus réactionnels ultrarapides. Cette méthode pompesonde permet d'une part une identification précise et d'autre part une caractérisation structurale de ces différentes espèces labiles. Nous rappelons brièvement dans cet article le principe de la spectrométrie Raman de résonance résolue dans le temps puis donnons une description de l'installation de spectrométrie Raman de résonance picoseconde développée au laboratoire. Enfin, un exemple d'étude de structure d'un état excité $\mathrm{S}_{1}$ de courte durée de vie $\left(\mathrm{t}_{1 / 2} \sim 10 \mathrm{ps}\right)$ impliqué dans un processus de photoréduction ultrarapide est donné à titre d'illustration.
\end{abstract}

\section{INTRODUCTION}

Une meilleure compréhension des processus photophysiques et de la réactivité photochimique en phase condensée nécessite la caractérisation spectroscopique en temps réel des différents intermédiaires réactionnels impliqués dans ces processus (états excités, ions, radicaux...). La plupart des travaux effectués dans ce domaine s'intéressent aux aspects cinétiques des réactions chimiques mais peu à la caractérisation de la conformation des différentes espèces labiles intermédiaires participant à celles-ci. La géométrie et la distribution électronique d'une molécule sont cependant des paramètres essentiels qui gouvernent sa réactivité. Au cours de la réaction, les réactifs évoluent sur des surfaces de potentiel définies et subissent des distorsions structurales selon des coordonnées spécifiques qui constituent le chemin réactionnel. En solution, celles-ci peuvent inclure des interactions de type soluté-solvant. Caractériser les coordonnées de réaction et les interactions entre réactifs et solvant, et obtenir des informations structurales sur les intermédiaires réactionnels, constituent des aspects fondamentaux de la recherche sur les mécanismes réactionnels en solution, avec l'objectif ultime de trouver, si possible, des moyens de contrôle des réactions chimiques.

La structure à l'équilibre d'une molécule stable à l'état fondamental peut généralement être déterminée très précisément par les techniques de diffraction et différentes techniques spectroscopiques classiques. Par contre, caractériser la structure d'états électroniques excités ou d'intermédiaires réactionnels reste aujourd'hui difficile du fait de leur courte durée de vie. Les spectrométries d'absorption et d'émission électroniques transitoires permettent certes de suivre en temps réel l'évolution cinétique d'intermédiaires réactionnels en cours de réaction avec une excellente résolution temporelle mais renseignent peu sur la structure de ces espèces en solution. Par ailleurs, les efforts menés actuellement pour développer des sources $\mathrm{X}$ pulsées et mettre au point des techniques de diffraction résolues en temps permettront certainement dans un avenir proche d'accéder à la structure instantanée de systèmes en évolution avec une résolution temporelle sub-picoseconde. Cependant, la difficulté d'obtenir des informations pour des espèces fortement diluées en solution restera probablement une limitation majeure de ces techniques. Les spectrométries vibrationnelles résolues en temps, notamment la diffusion Raman de résonance picoseconde, restent à ce jour inégalées pour leur aptitude à sonder en temps réel l'évolution structurale d'espèces chimiques transitoires en phase condensée et à caractériser à l'échelle moléculaire les interactions spécifiques soluté-solvant. En effet, les spectres de vibration, véritables empreintes digitales des molécules, permettent une identification précise des différentes espèces instables. De plus, la spectrométrie Raman de résonance résolue dans le temps permet une description détaillée des modifications de géométrie et de distribution électronique subies par les molécules tout au long du chemin réactionnel. Cette description n'est pas triviale du fait des difficultés rencontrées pour interpréter les spectres de vibration en terme de variations de structure pour des molécules peu symétriques et/ou 
comportant un nombre d'atomes important. Cette contrainte sévère restreint souvent l'utilisation de la spectrométrie Raman de résonance résolue dans le temps à des fins d'analyse structurale aux molécules de petite taille présentant un degré de symétrie élevé. Dans ce cas, une description qualitative des variations de géométrie des molécules peut généralement être faite directement à partir des spectres de vibration. Toujours dans le cadre restrictif de molécules de taille réduite (une vingtaine d'atomes), il est possible de parvenir à une description quantitative des structures des états excités et intermédiaires réactionnels par l'utilisation de méthodes de chimie quantique notamment les méthodes $a b$ initio. Dans ce cas, la comparaison des spectres de vibration expérimentaux et calculés permet de valider les structures optimisées par le calcul.

\section{PRINCIPE DE LA SPECTROMETRIE RAMAN DE RESONANCE RESOLUE EN TEMPS}

Le principe de l'étude des états excités et des intermédiaires réactionnels par spectrométrie Raman de résonance résolue dans le temps est illustré sur la figure 1. Il fait appel à la méthode pompe-sonde et nécessite l'utilisation de deux impulsions lasers décalées dans le temps. L'échantillon est excité à l'instant $\mathrm{t}=0$ par une impulsion laser de perturbation, $h v_{p}$, accordée sur une bande d'absorption de l'un des réactifs. Le spectre Raman du milieu perturbé est ensuite enregistré au moyen d'une seconde impulsion laser, $h v_{s}$, retardée d'un retard $\Delta t$ par rapport à la première. La description cinétique du système s'effectue en faisant varier le retard $\Delta t$ entre les impulsions de pompe et de sonde. Pour être en mesure de détecter une espèce transitoire généralement formée en très faible concentration, il est nécessaire de se placer en condition de résonance, c'est-à-dire choisir la longueur d'onde de sonde en accord avec une transition électronique de l'espèce transitoire. Dans ce cas, une très forte exaltation de l'intensité Raman est observée pour les modes de vibration qui donnent lieu à une activité vibronique dans le spectre électronique, c'est-à-dire essentiellement les modes totalement symétriques du chromophore impliqué dans la transition résonnante. $\mathrm{Si}$ la réaction met en jeu une succession d'intermédiaires réactionnels, leur caractérisation nécessite d'ajuster, pour chacun d'eux à tour de rôle, la longueur d'onde de sonde et le retard entre perturbation et sonde en fonction de leurs propriétés d'absorption et de leurs comportements cinétiques respectifs.

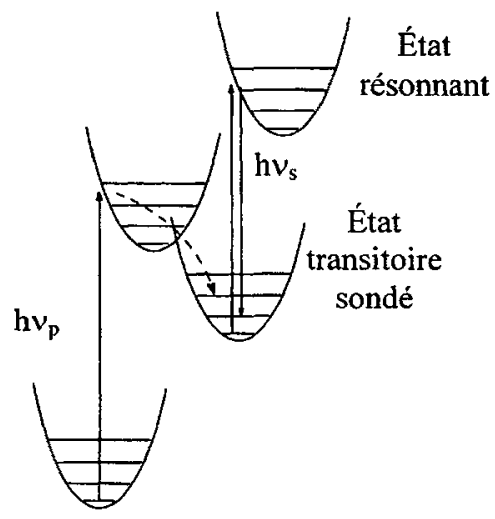

Figure 1: Principe de l'étude d'un état transitoire par spectrométrie Raman de résonance résolue en temps

\section{DISPOSITIF EXPERIMENTAL DE SPECTROMETRIE RAMAN PICOSECONDE}

L'installation de spectrométrie Raman de résonance picoseconde développée au LASIR comporte une source laser picoseconde générant les impulsions de pompe et de sonde, une ligne à retard optique 
permettant de les décaler temporellement, un spectrographe mono-étage précédé d'un filtre optique de type notch chargé de réduire de plusieurs ordres de grandeur l'intensité de la lumière diffusée à la fréquence du laser entrant dans le spectromètre et un détecteur multicanal à bas niveau lumineux utilisant une matrice $C C D$ amincie illuminée face arrière. L'originalité de ce montage réside dans l'utilisation d'une source laser à base de saphir dopé titane (Ti-Sa). L'avantage des sources laser amplifiées utilisant de ce matériaux comparativement aux sources à colorant n'est plus à démontrer (énergie 100 fois supérieure, stabilité, fiabilité...). Cependant ces sources sont restées à ce jour principalement utilisées pour la génération et l'amplification d'impulsions femtoseconde. Ceci tient à la technique d'amplification à dérive de fréquence mise en cuvre dans ces amplificateurs. La relation de Fourier qui existe entre la durée et le spectre d'une impulsion ne permet pas l'utilisation d'impulsion femtoseconde en spectrométrie Raman. En effet, la largeur spectrale d'une impulsion femtoseconde est incompatible avec la résolution spectrale requise en spectrométrie de vibration. C'est pour cette raison que l'utilisation de la spectrométrie Raman de résonance résolue dans le temps est généralement limitée au régime picoseconde. Nous avons modifié une source Ti-Sa femtoseconde afin de pouvoir la faire fonctionner en régime picoseconde. L'adoption d'étireurs d'impulsions très dispersifs nous a permis d'amplifier des impulsions de 3 ps (spectre de $\sim 4 \mathrm{~cm}^{-1}$ à $800 \mathrm{~nm}$ ) à des niveaux d'énergie proches du millijoule sans dégradation notable du spectre de l'impulsion (fonctionnement proche de la limite de Fourier). Cette source est accordable sur le domaine $740-850 \mathrm{~nm}$. La $3^{\text {eme }}$ et la $2^{\text {eme }}$ harmonique de ce laser peuvent être utilisées comme pompe et comme sonde. Cependant, dans ce cas, l'accordabilité est réduite à un domaine spectral limité et on ne peut pas faire varier indépendamment les longueurs d'onde de pompe et de sonde. Le développement d'un générateur et d'un amplificateur paramétrique optique affiné, pompé par la seconde harmonique du laser Ti-Sa $(400 \mathrm{~nm})$ a permis de pallier ces défauts et d'étendre la gamme spectrale couverte au domaine $460-675 \mathrm{~nm}$ [1]. Le schéma de principe de cette source est donné sur la figure 2 . La résolution temporelle (intercorrélation pompe-sonde) du dispositif expérimental est de l'ordre de $3.5 \mathrm{ps}$.

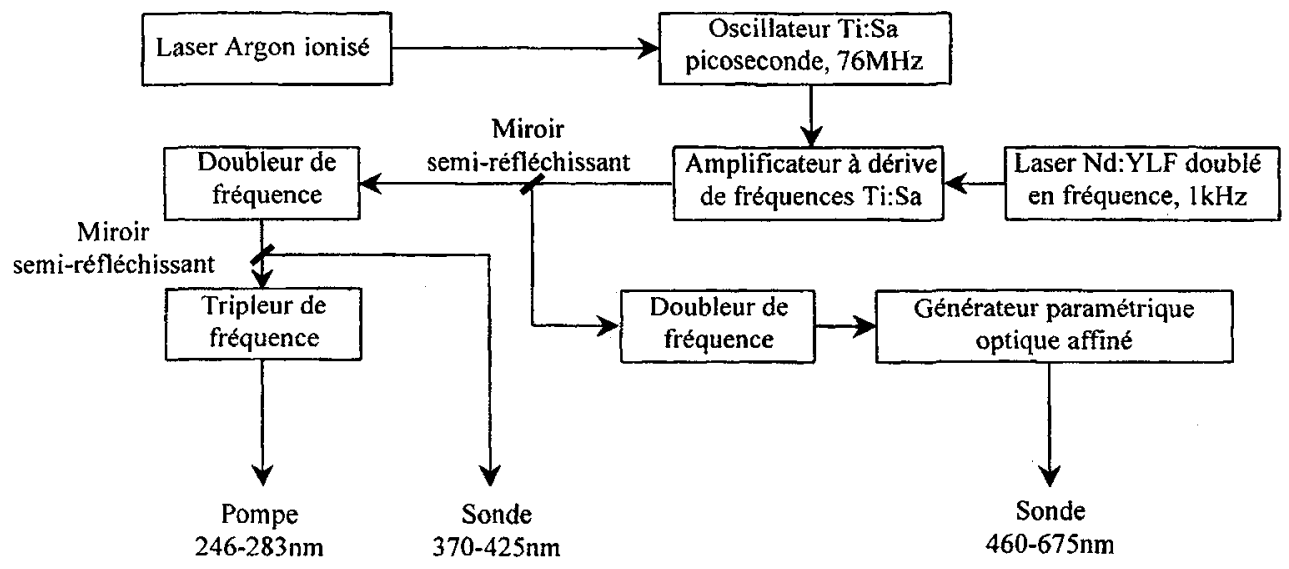

Figure 2 : Schéma de principe de la source laser picoseconde développée pour la spectrométrie Raman de résonance transitoire

\section{DETERMINATION DE LA STRUCTURE D'UN ETAT EXCITE $S_{1}$}

La molécule de 4,4'-bipyridine (44BPY) est photoréduite par les alcools à l'état $\mathrm{S}_{1}\left(\mathrm{n} \pi^{*}\right)$ et à l'état $\mathrm{T}_{1}$ $\left(\pi \pi^{*}\right)$ pour donner le radical $\mathrm{N}$-hydro $(44 \mathrm{BPYH})$. Tandis que la vitesse de réaction à l'état $\mathrm{T}_{1}$ $\left(\mathrm{k}_{\mathrm{q}}=10^{5} \mathrm{M}^{-1} \mathrm{~s}^{-1}\right)$ est comparable à celle généralement observée pour un transfert d'atome d'hydrogène [2], celle à l'état $S_{1}$, supérieure par cinq ordres de grandeur, semble plutôt typique des vitesses de transfert 
d'électron. Il a été possible de vérifier la nature des processus en utilisant le fait que, grâce à la spécificité spectrale élevée de la spectrométrie Raman, le radical hydrogéné 44BPYH et son homologue deutérié 44BPYD présentent des signatures distinctes. Des expériences effectuées en présence de méthanol deutérié partiellement soit en position hydroxyle, soit sur le groupement méthyle, révèlent que les réactions résultent dans les deux états excités du même processus de transfert d'un atome d'hydrogène du groupement méthyle de l'alcool. La vitesse exceptionnellement élevée du transfert d'atome d'hydrogène à l'état $\mathrm{S}_{1}\left(\mathrm{k} \sim 5.10^{10} \mathrm{~s}^{-1}\right)$ est surprenante et ne peut s'expliquer à partir des seules considérations thermodynamiques. Ce point particulier a motivé la recherche d'informations sur la structure de l'état $S_{1}$ et sur celle du radical [1]. Les spectres Raman de l'état $S_{1}$ ont été enregistrés en résonance avec deux transitions électroniques situées vers 380 et $580 \mathrm{~nm}, 5 \mathrm{ps}$ après perturbation par une impulsion de pompe (Figure 3a). Les fréquences observées contiennent potentiellement des informations sur la structure de cet état. Cependant dans le cas de molécules aromatiques, les vibrations ne sont pas localisées sur une coordonnée interne spécifique (liaison, angle), mais correspondent plutôt à des modes normaux distribués sur un ensemble de coordonnées, avec une distribution différente dans les états fondamental et excités. L'interprétation quantitative des données vibrationnelles transitoires en terme de structure a par conséquent nécessité l'assistance de calculs quantiques. Dans le cas de l'état $\mathrm{S}_{1}$ de la 44BPY, nous avons pu montrer que sa structure est asymétrique (figure $3 \mathrm{~b}$ ) avec l'excitation localisée sur un seul des deux cycles pyridines [3]. La liaison intercycle est peu affectée et l'atome d'azote du cycle perturbé est situé hors du plan de celui-ci du fait d'un certain caractère $\mathrm{sp}^{3}$. Il existe une forte analogie entre cette structure et celle déterminée similairement pour le radical N-hydro [4]. L'essentiel de l'évolution structurale accompagnant le processus de photoréduction est accompli au cours de la transition de $S_{0}$ à $S_{1}$, le passage au radical s'effectuant ensuite sans réorganisation structurale notable et par conséquent avec une barrière d'activation du transfert d'hydrogène faible en accord avec la vitesse très élevée de celui-ci.
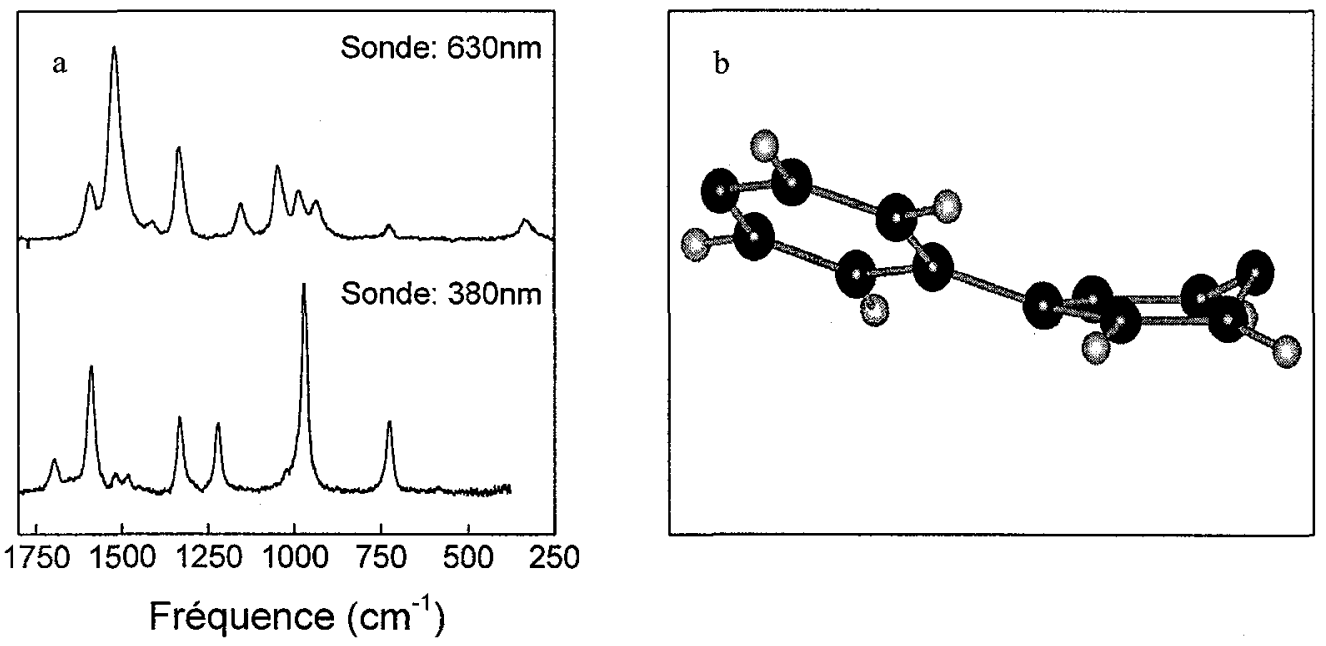

Figure 3: Spectres Raman de résonance de l'état excité $S_{1}$ de la 44BPY excités en résonance avec deux transitions électroniques distinctes (a) et structure ab initio optimisée correspondant (b)

\section{Références}

1. C. Didierjean, V. De Waele, G. Buntinx, O. Poizat, Chem. Phys. 237, 169 (1998)

2. O. Poizat, G. Buntinx, P. Valat, V. Wintgens, M. Bridoux, J. Phys. Chem. 97, 5905 (1993)

3. V. De Weale, G. Buntinx, O. Poizat, J. Chem. Phys. 110, 6353 (1999)

4. M. Castella-Ventura, E. Kassab, J. Raman Spectrosc. 29, 511 (1998) 